\title{
Kriminalprognose und Therapiebeurteilung
}

\author{
Hans-Ludwig Kröber ${ }^{1}$
}

(c) Springer-Verlag GmbH Deutschland, ein Teil von Springer Nature 2019

In der kriminalprognostischen Begutachtung treffen sich oftmals zwei Aufgaben: die Beurteilung der möglicherweise anhaltenden Gefährlichkeit einer Person und die Beurteilung der Therapie, welcher diese Person unterzogen wurde. Letzteres wird bisweilen vernachlässigt; wenn sich jemand trotz längerer Therapie nicht ändert, liegt es aus der Sicht mancher Gutachter stets an der Person, nicht aber an der Therapie. Tatsächlich aber können ungeeignete Therapien, oder auch die Konzentration auf bestimmte psychotherapeutische Verfahren statt auf psychoedukative Milieutherapie und Beziehungsarbeit, zu einer weiterhin schlechten Prognose führen.

In diesem Heft nun stehen zunächst die neuen „Empfehlungen“ für kriminalprognostische Gutachten im Vordergrund, beginnend mit dem umfänglichen juristischen Teil von Axel Boetticher, Matthias Koller et al., der dem psychiatrischen und psychologischen Leser die rechtlichen Rahmenbedingungen der kriminalprognostischen Begutachtung verdeutlicht. Der juristische Beitrag ist ebenso wie die nachfolgenden erfahrungswissenschaftlichen Empfehlungen für Prognosegutachten von Kröber, Rettenberger, Brettel und Stübner eine intensive Überarbeitung und schließlich Neufassung der 2006/2007 veröffentlichten „Mindestanforderungen für Prognosegutachten“ (Boetticher et al. 2007) einer Arbeitsgruppe von Bundesrichtern und weiteren Juristen, forensischen Psychiatern und Rechtspsychologen, die damals wie auch jetzt auf Initiative von Axel Boetticher zustande kam. Wie damals werden juristischer und psychiatrisch-psychologischer Teil parallel in der Neuen Zeitschrift für Strafrecht (NStZ) veröffentlicht, da sich die Empfehlungen gleichermaßen an die Juristen richten, die in der Strafrechtspflege tätig sind.

Es gab zunächst lebhafte Diskussionen, ob überhaupt eine Neufassung der Mindestanforderungen sinnvoll ist; manche befürchteten eine Anpassung an die Tendenzen zur

Prof. Dr. Hans-Ludwig Kröber

mail@hlkroeber.de

1 Zentrum für Forensisch-Psychiatrische Begutachtung (ZFPB), Berlin, Deutschland
Vereinfachung der Prognose durch eine völlige Dominanz der Nutzung standardisierter Instrumente und entsprechend gruppenstatistischer, nichtindividualisierter Aussagen. Das Gegenteil ist eingetreten; es hat eine notwenige Anpassung gegeben, an die veränderte Rechtslage, insbesondere im Vollzug und bei der Beendigung der Maßregeln, und es gibt bereits gegenüber 2006 eine veränderte Begutachtungsstrategie, die sehr viel mehr einen Abgleich zwischen den Fähigkeiten und Schwächen eines Probanden einerseits und des aktiv zu gestaltenden sozialen Empfangsraumes andererseits vornimmt. Es sind in den letzten 15 Jahren eben doch viele Strukturen der Nachsorge aufgebaut worden, nicht zuletzt forensische Ambulanzen, die durch kompetente Nachsorge eine frühere Entlassung trotz noch vorhandener Risiken ermöglichen. Zudem wird, anders als damals, im erfahrungswissenschaftlichen Teil das Verhältnis von individueller und gruppenstatistischer, zwischen idiographischer und nomothetischer Betrachtungsweise offensiv aufgegriffen und als konstruktives Wechselverhältnis verhandelt.

Der interdisziplinäre Arbeitskreis, aus dem die beiden Beiträge hervorgegangen sind, hat eingehend deren Bezeichnung diskutiert und den Begriff „Mindestanforderungen“ durch „Empfehlungen“ ersetzt. Seinerzeit war der Vorwurf erhoben worden, der Arbeitskreis maße sich unzulässig Befugnisse an, Standards zu dekretieren, als sei er der Bundesgerichtshof. Andererseits wurde der Begriff von Anwälten und Gerichten genutzt, um missliebige Gutachten mit dem Argument zu diskreditieren, sie entsprächen nicht einmal den mindesten Anforderungen. Dies war nicht ganz selten unbegründet, wurde oft gar nicht nachgewiesen oder mit der Nichtbeachtung einiger formaler Regeln begründet. Es geht in den erfahrungswissenschaftlichen Empfehlungen darum, eine bestimmte kriminalprognostische Denkweise zu verdeutlichen, die dann den gutachterlichen Aussagen zugrunde liegt. Es geht im juristischen Beitrag darum, den juristischen Raum auszuleuchten, in dem sich die Erfahrungswissenschaftler nun einmal bewegen und in den sie gezielt etwas an Aufklärung einbringen sollen.

Teil der kriminalprognostischen Begutachtung ist die Erfassung und Beurteilung des Therapieverlaufs. Dazu muss 
der oder die Sachverständige vertraut sein mit den möglichen und gängigen Therapieverfahren, ihrer möglichen Wirkstärke und mit den Rahmenbedingungen, unter denen Therapie im Straf- und im Maßregelvollzug stattfindet.

Eine direkte Verbindung zwischen Therapie und Prognoseforschung findet sich im Beitrag von Stephan Veismann et al. über die (insgesamt geringe) Rückfälligkeit der aus dem psychiatrischen Maßregelvollzug Hamburgs entlassenen Patienten und deren Varianz in Abhängigkeit von Behandlungsdauer, Behandlungsbedingungen und psychiatrischer Problematik. Die im Rahmen einer Vollerhebung einer Metropolregion erfassten 73 Patienten mit langer Katamnesedauer litten ganz überwiegend unter Schizophrenie, mit oder (seltener) ohne komorbiden Substanzmissbrauch.

Der nachfolgende Beitrag von Klaus Elsner widmet sich einer Tätergruppe, die bei Rückfallstudien zumeist die höchste einschlägige Rückfälligkeit aufweist, nämlich persönlichkeitsgestörte Sexualstraftäter. In deren Behandlung im Maßregelvollzug, SothA, Sicherungsverwahrung finden wir je nach Ort und Therapeut ein Nebeneinander unterschiedlicher Therapieformen. Hartnäckig hält sich insbesondere seit 20 Jahren und mehr eine klassischen Suchttherapien entsprechende Abstinenztherapie, die eng auf das deviante Verhalten fokussiert und es quasi örtlich betäuben will. Elsner verdeutlicht, dass die therapeutische Entwicklung weitergegangen ist und Therapieformen hervorgebracht hat, die nicht ausschließlich auf Vermeidung aus sind, sondern auf eine neue, fortschrittliche Selbstbestimmung.

Damit in engem Zusammenhang steht der Sachverhalt, dass der wichtigste Wirkfaktor einer Therapie darin besteht (oder bestehen kann), dass der Patient in der Begegnung mit dem Therapeuten Beziehungsfähigkeit entwickelt. Auf diese Problematik fokussiert der Beitrag von Maximilian Meyer et al., die empirisch die Qualität der Therapeut-PatientBeziehung erfasst haben, bei Patienten, die halt nicht freiwillig, sondern aufgrund strafrechtlichen Urteils in Therapie gelangt waren. Sie verdeutlichen, wie unterschiedliche Haltungen des Therapeuten nachweislich die Qualität der Beziehung beeinflussen. Dies beeinflusst damit auch unmittelbar die Chancen des Untergebrachten auf Entlassung und Rückfallfreiheit.

Der Beitrag von Lena C. Carl et al. geht noch einen Schritt weiter und versucht, zu einem Benchmarking der sozialtherapeutischen Abteilungen zu kommen; man könnte auch sagen, zu einem Anforderungsprofil für ,,best practice“ in der Straftäterbehandlung. Die Autoren haben sieben sozialtherapeutische Abteilungen für Sexualstraftäter in Bayern hinsichtlich ihrer strukturellen Rahmenbedingungen, der behandelten Täter und deren Rückfälligkeit vergli- chen. Die Varianz in der Rückfälligkeit hing nur zu einem geringen Teil davon ab, in welcher Abteilung die Behandlung erfolgt war. Es könnte dies bedeuten, dass die Struktur der SothA nicht sehr wichtig ist, es könnte aber ebenso auf eine große Ähnlichkeit der entscheidenden institutionellen Wirkfaktoren in den untersuchten Sozialtherapien verweisen.

Der Blick auf die Institutionen, die in der Rückfallverhinderung und Besserung der prognostischen Aussichten tätig werden, wird abgerundet durch den Bericht von Jörg-Uwe Schäfer, der als Leiter der Abteilung für Sicherungsverwahrte in der JVA Werl (140 Verwahrte!) in seinem Werkstattbericht die Diskrepanz zwischen Theorie und Praxis in der Straftäterbehandlung anschaulich beschreibt, wobei die leicht sarkastische Grundierung in der Schilderung der Widrigkeiten verdeutlicht, dass der Verfasser weiterhin entschlossen ist, an der Erreichung des Vollzugsziels zu arbeiten.

Es folgt ein freier Beitrag von Barbara Bergmann zum Einfluss der Berufs- und Lebenserfahrung von Polizeibeamten im Vergleich mit Laien bei der Beurteilung der künftigen Delinquenzgefährdung von straffälligen Jugendlichen, ob es sich hier also um künftige Intensivtäter handelt, die einer besonderen Erfassung und Behandlung bedürfen. Auch dies ist eine praxisrelevante Form der Kriminalprognose.

Es folgt im Heft wie gewohnt der Journal Club mit dem psychologischen Beitrag von Stjepan Curic über Korrelate zukünftiger Gewalthandlungen bei schizophren Erkrankten und dem kriminologischen Beitrag von Marleen Gräber und Barbara Horten zu der Thematik, ob die Zuwanderung von Flüchtlingen mitursächlich für die Zunahme der Gewaltkriminalität in Deutschland ist. Das Blitzlicht flankiert das Schwerpunktthema, indem es die kriminalprognostischen Schwierigkeiten bei der Begutachtung von älteren Herren beleuchtet, die den Eindruck zu erwecken versuchen, dass sie Straftäter im Ruhestand sind.

Und zum Schluss wieder forensische Reise-Empfehlungen im Kongresskalender.

\section{Hans-Ludwig Kröber}

Interessenkonflikt H.-L. Kröber gibt an, dass kein Interessenkonflikt besteht.

\section{Literatur}

Boetticher A, Kröber H-L, Müller-Isberner R, Böhm KM, MüllerMetz R, Wolf T (2007) Mindestanforderungen für Prognosegutachten. Forens Psychiatr Psychol Kriminol 1:90-100 